\title{
HNO-Notfälle \\ Die wichtigsten Erkrankungen im Überblick
}

Friedrich Bootz • Peter K. Plinkert • Hans-Peter Zenner

Notfallsituationen im Hals-Nasen-Ohren-Bereich sind relativ häufig. Um eine vitale Bedrohung des Patienten bzw. schwere bleibende Schäden abzuwenden, müssen Sie als Arzt schnell handeln - auch wenn Sie kein HNO-Experte sind. Orientierung geben die auffälligen Leitsymptome, wie z. B. Atemnot oder Blutungen. Sie grenzen die Diagnose der zugrundeliegendenErkrankung stark ein und erleichtern die rasche Auswahl der passenden Soforttherapie. Diese Übersichtsarbeit stellt die wichtigsten Notfälle an Hals, Nase und Ohren vor und skizziert, wie man Patienten optimal versorgt.

Einleitung HNO-Notfallsituationen erfordern häufig rasches ärztliches Handeln. Das gilt für beide Arten von Notfällen:

- HNO-Erkrankungen, die nicht vital bedrohlich sind, aber notfallmäßig therapeutische Maßnahmen erfordern, sowie

- Erkrankungen oder therapeutische Eingriffe im Kopf-Hals-Bereich, die zu einer vitalen Bedrohung (z.B. der Atmung) des Patienten und damit zu einer Notfallsituation führen.

Im Folgenden werden v.a. häufige Notfälle näher beschrieben sowie solche, die den Patienten vital gefährden. Für die übrigen Notfälle sei auf @ Tab. e 1 (online) verwiesen. Sie listet für jeden Notfall die wichtigsten Schritte auf, die Sie über die üblichen Notfallmaßnahmen hinaus ergreifen sollten.

\section{Respiratorische Notfälle}

Ursachen für Atemnot Eine der dramatischsten Notfallsituationen, mit denen der Arzt konfrontiert werden kann, ist die vitale Störung der Atemfunktion. Sie kann bedingt sein durch:

- Störungen der zentralen Atemregulation,

- eine mechanische Obstruktion der Atemwege,

- eine Behinderung der Ausdehnungsfähigkeit der Lunge (z.B. Pneumothorax),

- Störung des Gasaustausches und der Atemgaszusammensetzung.

Symptome Eine respiratorische Insuffizienz beginnt oft mit uncharakteristischen Symptomen wie Unruhe, Verwirrtheit und Tachykardie.

- Als Leitsymptom respiratorischer Notfälle gelten Dyspnoe und Zyanose. Diese ist v.a. an den Lippen zu erkennen.

Das Endstadium der respiratorischen Insuffizienz ist der Atemstillstand. Er ist gekennzeichnet durch fehlenden Atemstoß, fehlende Atembewegung, Zyanose und Eintreten des Kreislaufstillstands.

Erste Hinweise auf die Diagnose Anhand von Atemfrequenz und -geräusch lässt sich die Ursache der Atemnot bereits weiter eingrenzen.

- Eine Tachypnoe deutet auf eine periphere Atemstörung hin,

- eine Bradypnoe dagegen auf eine zentrale Atemstörung.

- Atmet der Patient forciert, blockiert wahrscheinlich ein mechanisches Hindernis die oberen Luftwege.

- Auch inspiratorische Atemgeräusche sind Hinweise auf eine Obstruktion der oberen Luftwege: Ein Stridor tritt bei Atemwegshindernissen im Bereich des Larynx und der Trachea auf.

- Auskultatorisches Pfeifen, Giemen und Brummen entstehen bei Obstruktionen im Bronchialbereich, z. B. beim Asthma bronchiale.

- Schnarchende Atemgeräusche deuten auf eine Obstruktion im Pharynx hin - z.B. durch Zurückfallen der Zunge.

Details zum Management von Atemnot finden sich in Lege artis 1/11 [1]. Daher beschränkt sich diese Übersicht auf mechanische Obstruktionen.

\section{Mechanische Obstruktion der oberen Atemwege}

$\nabla$

Ursache Für eine mechanische Obstruktion der Atemwege kommen verschiedene Gründe infrage:

- Tumoren,

- Entzündungen und Verletzungen im Pharynx-, Kehlkopf- oder Trachealbereich oder

- Aspiration von Fremdkörpern 
- Auch chronisch hyperplastische Tonsillen beim Kind können zu einer Obstruktion der Atemwege führen.

Blockade durch Tumor oder Entzündung Bei Entzündungen, Hyperplasien oder Tumoren erfolgt in Notsituationen ggf. die Sofortintubation - auch endoskopisch mit flexibler Optik oder starrem Rohr.

- Bei Tumoren, die in die Trachea eingewachsen sind, sollte zuerst ein dünnes starres Endoskop über die Enge hinweggeschoben werden. Dies erfolgt unter Absaugbereitschaft, da es zu starken Blutungen kommen kann!

- Danach wird die Stenose mit starren Endoskopen in aufsteigender Größe erweitert.

In seltenen Fällen gelingt die Intubation nicht. So können Tumoren des Larynx, Hypopharynx oder der Schilddrüse direkt zur Obstruktion führen.

- Dann kann eine Tracheotomie oder eine Koniotomie angezeigt sein.

Bei laryngektomierten bzw. tracheotomierten Patienten Nach Eingriffen an Kehlkopf oder Luftröhre besteht die Neigung zu Verborkung der Trachea und dadurch bedingter Obstruktion.

- In diesem Fall kann man versuchen, die Borke mit einer Zange, evtl. durch ein starres Bronchoskop (Würzburger Notfall-Bronchoskop), zu extrahieren.

- Die Trachea wird vorsichtig mit Kochsalzlösung angespült, die Absaugung erfolgt mit einem Absaugkatheter.

Rekurrensparese Die Symptome der Rekurrensparese imitieren eine mechanische Obstruktion. Sie können durch Krankheiten, aber auch durch Komplikationen nach Schilddrüsen-Operationen oder Tumoren bedingt sein.

Denken Sie an eine mögliche Parese des N. laryngeus recurrens bei Patienten mit OP-Narbe im Bereich der Tyroidea.

- Die einseitige Parese zeigt sich zumeist in Heiserkeit, da die Stimmlippen nicht mehr voll bewegt werden können.

$\triangleright$ Cave: Die Heiserkeit kann aber auch komplett fehlen!

$\triangleright$ In der Regel leidet der Patient nur unter Belastungsdyspnoe.

- Bei einer doppelseitigen Rekurrenslähmung besteht eine Immobilisierung beider Stimmlippen in Medianstellung oder Paramedianstellung.

$\triangleright$ Die Stimme ist häufig unauffällig.

$\triangleright$ In Ruhe besteht meist eine Atemnot mit inspiratorischem Stridor, eine Belastung ist häufig nicht mehr möglich.

Bei vitaler Atemnot durch eine Rekurrensparese müssen Sie intubieren!
Angioödeme Es gibt verschiedene Typen von Angioödemen im Mund-Hals-Bereich. In über 90\% der Fälle tritt jedoch das Quincke-Ödem als Form der rezidivierenden Schleimhautschwellung auf.

- Mediatoren, die dieses Ödem hervorrufen, sind vor allem Histamin und vasoaktive Substanzen, wie z.B. ACE-Hemmer.

Nicht steroidale Antiphlogistika sind zur Therapie beim Angioödem weniger geeignet: Ein Drittel der Patienten erleidet nach dem erstmaligen Auftreten trotz der Therapie ein erneutes Ödem - obgleich keine Allergie gegenüber diesen Substanzen vorliegt. Mittel der Wahl ist daher

- die intravenöse Gabe von Glukokortikoiden wie Prednisolon ${ }^{\circledR}$ (250-1000 mg) und

- Antihistaminika,z.B. Fenistil ${ }^{\circledR}$-Injektionslösung (1 Brechampulle à $4 \mathrm{ml}, 1$ - bis 2-mal tgl.).

- Beim ACE-Hemmer-induzierten Ödem muss zudem das entsprechende Medikament abgesetzt werden.

Erwägen Sie immer, Angioödem-Patienten stationär zu überwachen und mögliche allergische Ursache abzuklären.

\section{Fremdkörper}

\section{Trachealfremdkörper}

$\nabla$

Meist sind Kinder betroffen Fremdkörper gelangen zumeist bei Kindern in die Luftröhre durch Aspiration von Spielzeug oder kleinen Nahrungsmittelteilen. Besonders gefährlich sind Erdnüsse.

Trachealfremdkörper bergen die akute Gefahr zu ersticken. Grund ist meist ein fremdkörperinduziertes Ödem und nicht der Fremdkörper selbst!

Versorgung des Patienten Patienten müssen daher unter ärztlicher Begleitung in die Klinik transportiert werden - bei Zyanose mit Sauerstoffmaske.

- In der Klinik beatmet man den Patienten dann tracheoskopisch, indem man das Endoskop an Ambubeutel, Beatmungs- oder Narkosegerät anschließt.

- Die Extraktion des Fremdkörpers erfolgt durch das starre Bronchoskop.

\section{Mund- und Pharynxfremdkörper}

$\nabla$

Ursache und Symptome Bei Fremdkörpern im Mund und Rachenbereich handelt es sich in der Regel um verschluckte Gräten, Nadeln oder Knochensplitter. In den meisten Fällen stecken diese in einer Gaumenmandel, seltener im Zungen- 
grund oder in der Vallecula epiglottica. Charakteristisch sind der spontan auftretende Schmerz und ein gesteigerter Würgereiz. Beides erschwert häufig die direkte Inspektion.

Inspektion Das Aufspüren eines Fremdkörpers in Mund und Pharynx ist nicht immer einfach.

- Die Lokalisation des Spontan- und Schluckschmerzes ist oft irreführend: Patienten fühlen den Schmerz in der Regel tiefer, als der Fremdkörper tatsächlich sitzt.

- Achtung: Besonders Gräten werden bei der einfachen Spiegeluntersuchung leicht übersehen, da sie manchmal sehr tief einspießen und dann kaum noch sichtbar sind.

Es empfiehlt sich daher eine indirekte Spiegeluntersuchung in Oberflächenanästhesie unter $\mathrm{Zu}$ hilfenahme von Untersuchungsmikroskop oder Lupenendoskop.

- Gelegentlich kann man den Sitz des Fremdkörpers auch durch sorgfältige Palpation herausfinden.

Eine Röntgenuntersuchung ist dagegen nur bei metallischen Fremdkörpern hilfreich.

Therapie Fremdkörper in Mundhöhle und Rachen müssen unter allen Umständen entfernt werden! Ein Abwarten ist nur zulässig, wenn der Patient seinen Schluckschmerz lediglich noch als nachlassenden Druck ohne bestimmte Lokalisation beschreibt - man also annehmen kann, dass der Fremdkörper von selbst abgegangen ist und nur noch die Schleimhautläsion Schmerzen verursacht.

- Bei tief in Richtung der Halsgefäße eingespießten Fremdkörpern ist eine Halseröffnung von außen unumgänglich.

- Eine Wandperforation mit schwerer parapharyngealer Entzündung erfordert

$\triangleright$ eine hoch dosierte intravenöse Antibiose (z.B. Claforan ${ }^{\circledR}$, 3- bis 6-mal 2g/d; Gerneb$\operatorname{cin}^{\circledR}$, 3-mal 40-80 mg/d; Clont ${ }^{\circledR}, 1,5-2 \mathrm{~g} / \mathrm{d}$; Augmentan $^{\circledR}, 3-$ mal 2,2 g/d) sowie

$\triangleright$ einen operativen Eingriff von außen mit Inzision und Drainage des Spatium parapharyngeum.

\section{Akute Schluckstörungen}

\section{Verbrühungen des Mund-Rachen-Raums}

$\nabla$

Ursache Verbrühungen entstehen im Wesentlichen durch das Trinken kochend heißer Getränke und kommen fast nur bei kleinen Kindern vor. Das Einatmen von Wasserdampf, z. B. durch Inhalatoren, schädigt dagegen eher die unteren Luftwege.

- Etwa 2-3 h nach dem Ereignis ist mit Heiserkeit und Atemnot zu rechnen, wenn sich die Verbrühung nicht auf die Mundhöhle beschränkt.
Therapie Heißes Wasser zerstört normalerweise nur die oberen Schleimhautschichten. Die tieferen Zellschichten bleiben intakt. Verbrühungen haben daher eine gute Prognose.

- Da die Verletzungen schmerzhaft sind und sich infizieren können, sind Schmerzmittel und eine antibiotische Abdeckung immer angezeigt,

- bei Atemnot (2-3h abwarten!) auch Steroide.

- Schwere Verbrühungen erfordern eine stationäre Behandlung.

- Hochgradiger Stridor zwingt zur Intubation, gegebenenfalls auch zur Tracheotomie.

\section{Verätzungen des Mund-Rachen-Raums}

Ursache Deutlich schwerer als Verbrühungen verlaufen Verätzungen, denn sie schädigen auch tiefergelegene Gewebeschichten. Verätzungen entstehen durch Ingestion von Säuren oder Laugen und ziehen in der Regel fast ausschließlich die Speisewege in Mitleidenschaft. Wiederum sind häufig Kinder betroffen, klassischerweise werden Haushaltsreiniger u.ä. geschluckt.

Ausmaß der Schäden Schädigungen bleiben auf die Mundhöhle beschränkt, wenn die ätzende Flüssigkeit infolge starker Schmerzen sofort wieder ausgespuckt wurde. Eine stärkere Schleimhautschädigung manifestiert sich durch ein Uvulaödem und Ätzschorfe, vorwiegend auf den Gaumenmandeln und dem Gaumensegel.

Ein Schluck einer starken Säure oder Lauge (bei Kindern 5-15 ml, bei Erwachsenen ca. $20 \mathrm{ml}$ ) kann bereits Lebensgefahr bedeuten. Es drohen Schock, Nierenversagen, Ösophagus- und Magenperforation sowie Arrosionsblutungen.

Sofortmaßnahmen Als erstes hilft es, den Patienten den Mund mit Wasser ausspülen zu lassen. Dies kann Progredienz z.T. verhüten. Das Wasser darf dabei nicht geschluckt werden!

- Kann man das Schlucken eines Ätzmittels nicht sicher ausschließen, ist bei Ätzspuren in der Mundhöhle und im Rachen eine endoskopische Kontrolle des Hypopharynx und des Ösophagus, ggf. auch eine Gastroskopie indiziert.

Weitere Behandlung Bei reinen Mundhöhlenverätzungen genügen Antibiotika (z.B. Amoxicillin, 3-6g/d) und Analgetika (z.B. Tramal ${ }^{\circledR}, 20$ Tropfen; bei sehr starken Schmerzen: Capros $^{\circledR}$ Tropfen, 20 mg, oder 0,5-1 Amp. Dipidolor ${ }^{\circledR}$ i.v.).

- Bei tief greifenden Verätzungen ist eine Narbenprophylaxe mit Steroiden indiziert (ab dem 12.-14. Tag $1 \mathrm{mg}$ Prednisolonäquivalent/ kg KG tgl. für 4 Wochen).

- Bei Spätschäden durch Narben sind Narbenexzisionen, ggf. auch Spalthaut- oder Schleimhauttransplantationen erforderlich. 


\section{Ösophagusverätzungen}

Symptome Aufgrund der kurzen Passagezeit können Ätzspuren in Mund und Rachen gering sein, während die Speiseröhre schwerste Verätzungen aufweist. Denken Sie an eine Ösophagusverätzung bei

- retrosternalen Schmerzen,

- Hypersalivation,

- Übelkeit, Brechreiz und Erbrechen

Sie stehen im Vordergrund der Symptomatik.

- Bei Larynxbeteiligung hört man einen Stridor.

- Kardiopulmonale Dysregulation, Schock und Nierenversagen sind möglich.

Typische Schäden durch Ätzmittel Laugen und Säuren wirken unterschiedlich. Anhand der Gewebeschäden lässt sich so die Art der Verätzung schon abschätzen.

- Laugen verursachen Kolliquationsnekrosen, d.h. sie verflüssigen das Gewebe. Dies geht meist mit tiefgreifender Wandschädigung, Rötung und sulziger Schwellung der Schleimhäute einher.

- Säuren dagegen führen zur Koagulationsnekrose - die Proteine im Gewebe gerinnen. Einige Substanzen hinterlassen dadurch charakteristische Spuren:

$\triangleright$ Salzsäure führt zu weißlichen Belägen,

$\triangleright$ Salpetersäure zu gelblichen und

$\triangleright$ Schwefelsäure zu schwärzlichen Nekrosen.

Sofortmaßnahmen Unabhängig davon, wie die Verätzung entstanden ist, steht bei der Therapie die akute Notfallbehandlung im Vordergrund. Je länger der Unfall zurück liegt, umso ausgeprägter können die Schäden sein. Daher muss eine Therapie sofort eingeleitet werden.

- Legen Sie 1-2 venöse Zugänge und verabreichen Sie darüber

- ein Analgetikum (z.B. Piritramid; Dipidolor ${ }^{\circledR,}$ $1 / 2$ Amp. langsam i.v.) und

- Prednisolon $2 \mathrm{mg} / \mathrm{kg}$ i.v. (z.B. Solu-Decortin ${ }^{\circledR}$ H) zur Vermeidung von Narbenstrikturen.

Cave Bei massiver Ätzmittelingestion sind Kortisonpräparate wegen der Perforationsgefahr kontraindiziert. Auch die Gabe von Bikarbonat hat wegen der Perforationsgefahr durch die $\mathrm{CO}_{2}$ Entwicklung zu unterbleiben!

- Danach folgt die Herz-Kreislauf-Stabilisierung, bei Larynxödem ggf. auch Intubation oder Tracheotomie.

Welche Substanz wurde geschluckt? Klären Sie, welche Substanz der Patient geschluckt hat. Rufen Sie die nächstgelegene Vergiftungszentrale an, um spezifische Hinweise zur weiteren Therapie zu erhalten.
- Neutralisationsmaßnahmen kommen meist zu spät, da die Ätzwirkung bereits nach ca. $60 \mathrm{sec}$ abgeschlossen ist.

$\triangleright$ Bei Laugen kann man jedoch einen Neutralisierungsversuch mit verdünntem Essig (2\%) oder Zitronensaft unternehmen.

$\triangleright$ Bei Säuren kann versucht werden, mit Wasser, Milch oder Antazida zu verdünnen bzw. zu neutralisieren.

Weitere Diagnose Röntgenaufnahmen von Thorax und Abdomen sind obligatorisch.

- Mithilfe von isotonischem Kontrastmittel lassen sich Perforationen des Ösophagus darstellen.

Um den Schweregrad der Verätzung zu ermitteln, führt man 6-24h nach der Verätzung eine Frühendoskopie mit flexibler Optik durch.

- Indikation zur Endoskopie sind sichere oder fragliche Ingestion bei gleichzeitigem Vorliegen eines der folgenden Symptome:

$\triangleright$ Ätzspuren in Mund und Rachen,

$\triangleright$ Hypersalivation,

$\triangleright$ Würge- und Brechreiz

$\triangleright$ retrosternale und / oder epigastrische Schmerzen

Bei Perforationsverdacht ist die Endoskopie kontraindiziert!

Aufgabe der Frühendoskopie ist die sichere Stadieneinteilung ( $\bullet$ Tab. 1). Von ihr hängt die Anschlusstherapie ab.

Anschlusstherapie bei schwereren Verätzungen Hat die Frühendoskopie eine Schädigung vom Grad 1b oder höher gezeigt, ist eine Weiterbehandlung nötig.

- Bereits im Rahmen der Endoskopie kann dann eine Magenspülung erfolgen, wenn die Verätzung nicht länger als 3-4 h zurückliegt.

- Um Narbenstrikturen zu vermeiden, gibt man sofort nach Endoskopie Prednisolon $2 \mathrm{mg} / \mathrm{kg}$ tgl. bis zur 4. Woche (vgl. Sofortmaßnahmen).

$\triangleright$ Bei erhöhter Perforationsgefahr beginnt man damit erst ab dem 12.-14. Tag.

$\triangleright$ Geben Sie kein Prednisolon bei ausgeprägter Magenbeteiligung!

Tab. 1 Stadieneinteilung bei Verätzung des Ösophagus (nach $[2,3]$ ).

\begin{tabular}{|l|l|}
\hline \multicolumn{2}{|c|}{ Stadieneinteilung bei Verätzungen des Ösophagus } \\
\hline Grad 1a & $\begin{array}{c}\text { örtlich und auf Mukosa begrenzte Verätzung mit Rötung } \\
\text { und Schwellung }\end{array}$ \\
\hline Grad 1b & örtlich begrenzte Verätzung, aber zirkuläre Läsionen \\
Gefahr der Stenosierung
\end{tabular}


- Zur Reduktion weiterer ätzender Belastungen des Ösophagus kann man $\mathrm{H}_{2}$-Antagonisten (z. B. Ranitidin i.v.) verabreichen. Das mindert die Produktion von Magensäure.

- Damit sich die verletzte Speiseröhre nicht entzündet, erhält der Patient eine antibiotische Therapie. Geeignet dafür sind

$\triangleright$ Amoxicillin plus Clavulansäure i.v. (z.B. Augmentan $^{\circledR}$, 3-mal 1,2-2,2 g/d).

$\triangleright$ Bei Penicillin-Allergie: Clindamycin (z.B. Sobelin $^{\circledR}$, 3- bis 4-mal 300-600 mg/d i.v.), alternativ Cotrimoxazol (z.B. Eusaprim ${ }^{\circledR}$ oder Cotrim-ratiopharm ${ }^{\circledR}$ Ampullen SF, 2-mal 2 Amp. tgl.).

Ernährung des Patienten Feste Kost sollte die verletzte Speiseröhre in den ersten Wochen nicht passieren.

- Der Patient wird daher zunächst parenteral ernährt,

> später erhält er flüssige oder breiige Kost.

In Abhängigkeit vom Lokalbefund kann man ab Grad 1b schon während der Frühendoskopie eine Magensonde legen. Dies beugt späteren Strikturen vor. Nach 2-4 Wochen kann die Sonde entfernt werden.

Verlaufskontrolle Verätzungen können zu Strikturen und Stenosen des Ösophagus führen. Daher müssen betroffene Patienten regelmäßig Röntgenkontrollen mit Breischluck erhalten.

- Diese beginnen bei Hinweis auf Strikturbildung ab 12.-15. Tag, bei Bedarf erfolgt eine Frühbougierung der Stenose.

- Bei komplikationslosem Verlauf führt man neben der Röntgenkontrolle im Zweifel eine Kontrollendoskopie in der 3.-4. Woche nach der Verätzung durch. Sie dient zum Ausschluss von Strikturen vor Ende der Kortisontherapie.

\section{Ösophagusfremdkörper}

Ursache Auch Fremdkörper im Ösophagus können den Schluckvorgang behindern. Sie sitzen meist in der 1. Etage des Ösophagus.

- Bei Gebissträgern handelt es sich häufig um größere Fleischbrocken, da die Gaumenplatte das Gefühl für die Größe von Nahrungsbrocken einschränkt.

- Kinder verschlucken meist Geldmünzen oder Spielzeugteile.

Fremdkörper in den tieferen Ösophagusabschnitten sind selten. Sofern größere Nahrungsbrocken in den unteren Ösophagusabschnitten hängen bleiben, dann meist aufgrund einer Ösophagusstenose oder (selten) aufgrund eines Karzinoms.
Gut zu wissen Kleine Kinder, bei denen das Verschlucken des Fremdkörpers nicht beobachtet worden ist, fallen dadurch auf, dass sie plötzlich die Nahrung verweigern, ohne dass eine andere Erklärung gegeben werden kann. Oft sind Patienten sehr beunruhigt, wenn sie beim Trinken aus einer defekten Flasche versehentlich einen Glassplitter verschluckt haben. Solche Splitter setzen sich jedoch praktisch nie fest oder verursachen ernsthafte Verletzungen. Gefährlich sind dagegen Glassplitter, die mit gekauter Nahrung verschluckt werden. Sie sind in den Speisebrei eingehüllt und können sich während des Schluckens nicht in die Längsachse des Speiseweges drehen. Dasselbe gilt für Gräten, Nadeln, Nägel etc.

Standardtherapie Mittel der Wahl zur Fremdkörperextraktion ist die sofortige Ösophagoskopie mit starrem Endoskop. Wenn nötig und möglich, kann man den Fremdkörper mit endoskopischen Zerkleinerungszangen oder (bei geeigneten Nahrungsmittelfremdkörpern, z.B. Fleisch) durch Argon-Plasma-Koagulation zerlegen.

Schon bei berechtigtem Verdacht auf Fremdkörper ist eine Ösophagoskopie erforderlich. Dann sollte jedoch ein flexibles Endoskop zum Einsatz kommen.

So nicht! Versuche, einen Fremdkörper durch Essenlassen von Kartoffelbrei oder Sauerkraut weiter nach unten zu befördern, sind kontraindiziert. Zu warnen ist auch vor blinden Extraktionsversuchen mit sogenannten Münzenfängern oder vor dem Versuch, Fremdkörper blind mit Sonden in den Magen zu stoßen. Sie bringen den Patienten in akute Lebensgefahr.

Zervikale Ösophagotomie Falls Zerkleinerung und Fremdkörperentfernung erfolglos bleiben, kann in seltenen Fällen eine zervikale Ösophagotomie oder bei tief sitzenden Fremdkörpern auch eine Thorakotomie erforderlich werden. Bei Perforation sind ein Übernähen des Wanddefektes und eine hoch dosierte antibiotische Abdeckung erforderlich.

Prognose Kleine Fremdkörper gelangen nach anfänglichem Steckenbleiben oft spontan in den Magen und werden dann ausgeschieden (Stuhlkontrollen, ggf. Röntgenkontrollen). Wenn Fremdkörper schnell und komplikationslos zu entfernen sind, ergeben sich meist keine langfristigen Probleme. Bei längerer Liegedauer kann es infolge primärer Wandverletzung oder sekundärer Drucknekrose zur Mediastinitis kommen. 


\section{Blutungen im Kopf-Hals-Bereich}

\section{Nasenbluten (Epistaxis)}

Ursache Nasenbluten ist nicht immer harmlos. Es kann als sogenanntes „unstillbares“ Nasenbluten zu lebensbedrohlichen Zuständen führen und ist dann nur durch ein rasches Eingreifen zu beherrschen. Unterschieden werden

- lokale Blutungsquellen (z. B. Läsionen am Locus Kiesselbachi, Tumoren, Traumata, Rhinitis sicca anterior, Septumperforation),

- systemische Blutungsursachen (z.B. arterielle Hypertonie, Arteriosklerose, hämorrhagische Diathese, Markumarisierung, Infektionskrankeiten) oder

• Unfälle.

Therapie Bei schweren Blutungen tamponiert man die Nase beidseitig mit einem Salbenstreifen $(1 \mathrm{mx} 1 \mathrm{~cm})$. Diesen legt man mäanderförmig von hinten nach vorn oder von oben nach unten in die Nasenhöhle. Alternativ eignen sich mit Salbe versehene Fingerlingstamponaden. Als Widerlager sollte die kontralaterale Seite gleichermaßen tamponiert werden, auch wenn sie nicht blutet. Die Tamponade ist außen an der Nase zu fixieren, um eine Aspiration zu vermeiden.

- Das genaue Vorgehen beschreibt der nachfolgende Beitrag auf $\bullet$ S. 316.

Bei persistierender Blutung aus den dorsalen Abschnitten der Nase ist zusätzlich eine hintere Nasentamponade mit beidseits gelegten Doppelballonkathetern erforderlich (z.B. Xomed Epistat ${ }^{\circledR}$; pneumatischer Nasentubus nach Masing, Rüsch ${ }^{\circledR}$ ). Diese schiebt man von vorn durch die Nasenhaupthöhle bis zum Nasopharynx ein und bläst sie auf (vor Einführen prüfen, ob Ballons aufblasbar und dicht sind!) Wegen der Gefahr z.T. beträchtlicher Schleimhautschäden sollten Doppelballonkatheter nach spätestens $4-5$ Tagen entfernt werden.

Stillt die Ballontamponade die Blutung nicht oder liegt eine extrem starke Blutung vor (z. B. bei einer Mittelgesichts-Rhinobasisfraktur), wird in Narkose eine Bellocq-Tamponade eingeführt.

\section{Mund- und Pharynxblutungen}

Ursache Schweren Mund- und Pharynxblutungen liegen häufig Gefäßarrosionen durch Tumoren oder Unfälle zugrunde.

Erstmaßnahme: Atmung ermöglichen Die Blutungen können schnell lebensbedrohlich sein und neben einer lokalen Blutstillung notfallmedizinische Maßnahmen erforderlich machen. Massiver Blutverlust kann zum hypovolämischen Schock (siehe unten) oder - durch Aspiration von frischem Blut oder Koagula - zur respiratorischen Insuffizienz mit Erstickungstod führen.
Daher muss man bei jedem Patienten

- zuerst die oberen Luftwege freimachen und/ oder schützen,

- frisches Blut absaugen und

- Koagel entfernen.

Es ist wichtig, bei Blutungen im Bereich der oberen Luftwege einen starken Sauger mit Saugrohr bereitzuhalten.

Vitalfunktion sichern Anhand des Schockindex (๑Tab. 2) lässt sich die kardiozirkulatorische Situation beurteilen. Man muss dann ggf. sofort damit beginnen, die vitalen Funktionen wiederherzustellen bzw. aufrecht zu erhalten.

In schwierigen Fällen Bei komatösen Patienten oder nach Aspiration empfiehlt sich die sofortige Intubation: Sie gewährleistet die Beatmung und schützt die Atemwege vor weiterer Aspiration. Bei schwieriger Intubation kann das Würzburger Notfall-Bronchoskop eingesetzt werden.

Blutstillung Um einen weiteren Blutverlust zu vermeiden, muss eine ausreichende Blutstillung erfolgen.

- Bei Gefäßarrosionsblutungen (bei Malignomen) ist eine Kompression bis zur endgültigen operativen Versorgung notwendig.

- In vielen Fällen von Tumorblutungen im HNOBereich ist die Intubation zur Verhinderung einer Aspiration empfehlenswert. Massive Blutungen im Pharynx- und Mundhöhlenbereich können dann vorübergehend durch Tamponaden gestillt werden.

- Kann die Blutungsquelle nicht lokalisiert werden, ist es oft notwendig, die in das Gebiet führenden Gefäße von außen am Hals zu unterbinden - in manchen Fällen sogar die A. carotis externa.

- Im Einzelfall kann eine Angiografie mit nachfolgender Embolisierung infrage kommen.

\section{Schocksituationen}

\section{Hypovolämischer Schock \\ $\nabla$}

Therapie Ist der Volumenverlust durch eine Blutung im Hals-Kopf-Bereich sehr groß, kann es zum hypovolämischen Schock kommen. Bringen Sie den Patienten in diesem Fall zuerst in die klassische Schocklage:

- Lagern Sie den Oberkörper des Patienten flach, während Sie die Beine etwas höher legen.

- Danach erfolgt umgehend eine Volumensubstitution durch große Punktionskanüle, primär mit Blutersatzmitteln wie

$\triangleright$ Oxygelatinederivate (Grenzdosis $2000 \mathrm{ml}$ ),

$\triangleright$ Hydroxyethylstärke (3\%-/6\%-/10\%-Lösung, Grenzdosis 20 bzw. 33 bzw. 66 ml $/ \mathrm{kg} \mathrm{KG} \mathrm{tgl.).}$ 
Tab. 2 nach [4]

Abb. 1 Notfallmaßnahmen beim anaphylaktischen Schock (mod. nach [2]). Als Ursache eines derartigen Schocks kommen allergische Reaktionen durch Insektenstiche, aber auch durch diagnostische Maßnahmen infrage, z. B. Allergietests oder Desensibilisierungstherapien.

\section{Der Schockindex}

Der Schockindex nach Allgöwer bezeichnet das Verhältnis von Herzfrequenz zu systolischem Blutdruck:

Schockindex $=H F\left[\mathrm{~min}^{-1}\right] / R R_{\text {syst. }}[\mathrm{mmHg}]$

- Der Normalwert liegt bei $60 / 120=0,5$; die Schwankungsbreite bei Gesunden zwischen 0,4-0,7.

- Werte $>1$ weisen auf einen Volumenverlust von mind. $30 \%$ hin.

Das entspricht ca. 1,5I bei Erwachsenen.

In Notfällen kann die Grenzdosis zur Überbrückung bis zur Transfusion überschritten werden.

Praxistipp Blutersatzmittel in flexiblen Kunststoffbehältern sind denen in Glasflaschen vorzuziehen, da eine Druckmanschette angelegt werden und so die Volumensubstitution schneller erfolgen kann.

Transfusion Blutverluste $>2500 \mathrm{ml}$ beim Erwachsenen müssen mit Blutkonserven substituiert werden. Dies ist jedoch auch abhängig vom Allgemeinzustand und Alter des Patienten. So müssen beim „Herzpatienten“ bereits nach geringen Blutverlusten Blutkonserven gegeben werden.

\section{Vorgehen beim anaphylaktischen Schock \\ 1. Patienten lagern \\ bei subakutem Verlauf: 0,3-0,5 mg Adrenalin i.m./s.c. \\ kutaner Allergentest als Ursache: $1 \mathrm{ml}$ ) oder \\ - Akrinor $^{\circledast}(0,5-1 \mathrm{ml}$ langsam i.v.). \\ Anaphylaktischer und anaphylaktoider Schock} Injektionsarm abbinden, weitere $0,3-0,5 \mathrm{mg}$ Adrenalin zur Umspritzung der Allergenapplikation

\section{Zugang legen}

bei subakutem Verlauf: $\mathrm{H}_{1}$-Antihistaminikum i.v. (z.B. Clemastin 2-4 mg) 0,3-0,5 mg Adrenalin i.m./s.c. alle 20 min

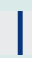

3. weiteres Vorgehen

falls RR nicht messbar: $0,1-0,5 \mathrm{mg}$ Adrenalin in $20 \mathrm{ml} 0,9 \% \mathrm{NaCl}$ i.v.

250-1000 mg wasserlösliches Kortikosteroid i.v.

$500 \mathrm{ml}$ Glukose-Elektrolytlösung (später ersetzt durch Volumenersatzmittel)

$\mathrm{O}_{2}$-Maskenbeatmung

Intubationsbereitschaft
Bis zur Bereitstellung des Blutes können initial Blutersatzstoffe (s. o.) eingesetzt werden.

- Bei dringendem Blutbedarf ist Spenderblut der Blutgruppe $0 \mathrm{Rh}^{-}$geeignet. Dennoch muss für eine nachfolgende blutgruppengleiche Transfusion Blut gekreuzt werden!

Die Gabe vasokonstriktorisch wirkender Sympathomimetika (Adrenalin) vor der Volumensubstitution sollte vermieden werden. Eine Intensivüberwachung ist notwendig.

\section{latrogener vasovagaler Schock}

Ursache Vasovagale Synkopen können durch iatrogene Manipulationen im Gehörgang, in der Nase und im Pharynx ausgelöst werden.

Symptome Beim vasovagalen Schock bestehen meist eine kurzfristige Bewusstlosigkeit, Kaltschweißigkeit, Blässe und Übelkeit.

Anders als beim hypovolämischen Schock tritt

Therapie Auch beim vasovagalen Schock empfiehlt sich die klassische Schocklagerung - also

- Oberkörper flach und Beine hoch.

Häufig genügt allein diese Maßnahme, dass sich der Patient rasch erholt. Führt sie nicht zum Erfolg, kann man den Blutdruck auch medikamentös steigern, entweder durch

- Parasympatholytika, wie z.B. Atropin (0,5-

Symptome Beim anaphylaktischen Schock ist die Hypotonie das Leitsymptom. Zusätzlich können Flush, Bronchospasmus, Übelkeit, Dyspnoe und Tachykardie auftreten. In fortgeschrittenen Stadien kann es zu Bradykardie, Bewusstlosigkeit, Atem- und Kreislaufstillstand kommen.

Therapie Wichtigster Schritt zu Beginn der Therapie ist,

- das auslösende Agens zu beseitigen.

Bei manifestem Schock ist das weitere Vorgehen auf das Sichern bzw. Wiederherstellen der Vitalfunktionen gerichtet:

- Injizieren Sie langsam Adrenalin i.v. (0,1-0,5 $\mathrm{mg}$, z. B. $0,5 \mathrm{ml}$ einer Lösung von Suprarenin ${ }^{\circledR}$ in $20 \mathrm{ml} \mathrm{NaCl}$ ).

- Anschließend gibt man Glukokortikoide i.v.:

$\triangleright$ Dexamethason $100 \mathrm{mg}$ oder

$\triangleright$ Prednisolon $1000 \mathrm{mg}$.

- Bei Deydratation des Patienten erfolgt ein Volumenersatz mit Plasmaexpander (z.B. beim vasovagalen Schock eine Bradykardie auf. 
Hydroxyethylstärke) oder Kristalloiden (z.B. Ringer-Lactat-Lösung, $100 \mathrm{ml}$ in $15 \mathrm{~min}$ ).

- Bei Atemstillstand müssen Sie intubieren und beatmen, bei Kreislaufstillstand zusätzlich kardiopulmonal reanimieren.

\section{Mittelgesichts- und Rhinobasisfrakturen}

Ursache Für Mittelgesichts- und Rhinobasisfrakturen sind in erster Linie Mechanismen direkter Gewalteinwirkung von Bedeutung. Verkehrsunfälle sind die häufigste Ursache. In 50\% der Fälle sind Mittelgesichts- und Rhinobasisfrakturen miteinander kombiniert.

Arten von Frakturen Die frontale Gewalteinwirkung kann zu Kieferfrakturen führen, die teilweise den von Le Fort beschriebenen Typen entsprechen. Sie kann aber auch als zentrale Mittelgesichtsfraktur in Erscheinung treten.

- Die wesentlichen Kriterien der Mittelgesichtsfrakturen vom Typ Le Fort I-III sind Störungen der Okklusion und insbesondere ein frontal offener Biss, der durch Abrutschen des Oberkiefers nach hinten und unten zustande kommt.

- Bei isolierten zentralen Mittelgesichtstrümmerfrakturen treten keine Okklusionsstörungen auf, da die hauptsächliche Gewalteinwirkung von frontal auf die Glabella trifft.

Sofortoperation Unter folgenden Umständen darf eine OP nicht aufgeschoben werden:

- bei ausgedehnten frischen Weichteilverletzungen mit Rhinobasisfraktur,

- bei Dura- und Hirnverletzungen und anhaltend starkem Liquorfluss,

- bei Eindringen von Fremdkörpern (Pfählung, Projektile) sowie

- bei traumatisch bedingter Kompression des N. opticus.

Besonderheit bei Sehnerv-Schädigung Eine Kompression des Sehnervs oder eine direkte Verletzung durch Frakturen bzw. Knochensplitterabsprengungen können zu einer rasch zunehmenden Sehverschlechterung und Erblindung führen.

- In diesen Fällen ist eine operative Dekompression des Sehnervs so zügig wie möglich angezeigt. Eingriffe jenseits der 12-h-Frist haben keine Erfolgsaussicht. Über die Indikation zur Dekompression entscheidet der Augenarzt.

Sind Frakturen am Dach des Optikuskanals vorhanden, ist die Dekompression mittels Kraniotomie durch den Neurochirurgen indiziert.

Aufgeschobene Dringlichkeit Nicht alle Patienten können oder müssen sofort operiert werden. Wenn keine Weichteilverletzungen vorliegen und dem Patienten aufgrund anästhesiologischer und neurochirurgischer Vorbehalte eine längere Narkose nicht zuzumuten ist, kann man die OP verschieben. Bei gesicherter Rhinobasisfraktur erfolgt zunächst eine Therapie mit einem liquorgängigen Antibiotikum.

- Innerhalb von 2-3 Wochen sollten jedoch alle Le-Fort-Frakturen und beeinträchtigenden Okklusionsstörungen versorgt werden. Sonst kann Bindegewebe in die Frakturspalten einwachsen und die knöcherne Ausheilung behindern. Hier droht eine Oberkieferpseudarthrose mit Restmobilität und entsprechender Beeinträchtigung des Kauvermögens.

- Die Versorgung isolierter Rhinobasisfrakturen kann man durchaus 6-8 Wochen aufschieben, wenn es die Allgemeinsituation des Patienten erfordert. Kommt es jedoch nach anfänglichem Sistieren erneut zu starker Rhinoliquorrhö oder zu einer aszendierenden Infektion, muss man die Rhinobasis ggf. rascher versorgen.

- Bei kombinierten Rhinobasis-GesichtsschädelFrakturen richtet sich der Operationszeitpunkt in der Regel nach der Gesichtsschädelfraktur.

Operationsmanagement Bei der chirurgischen Versorgung hat sich die Operationsplanung von innen nach außen bewährt. Falls eine Rhinobasisfraktur ( = innen) vorliegt, wird diese zuerst versorgt (I). Dann folgt der interorbitale Raum mit Siebbein, Nase, medialen Orbitawänden und kaudaler Stirn (II), zuletzt das übrige Mittelgesicht (= außen, III). Rhinobasis und interorbitaler Raum operiert der Rhinochirurg, das übrige Mittelgesicht der Kieferchirurg.

Fazit Die meisten HNO-Notfälle müssen rasch behandelt werden, um bleibende Schäden oder Lebensgefahr vom Patienten abzuwenden. Jeder Arzt sollte in diesen Fällen die grundlegenden Notfallmaßnahmen anwenden. Darüber hinaus ist es hilfreich, über die weiteren therapeutischen Schritte informiert zu sein. So können bereits die Grundlagen für eine zügige Weiterbehandlung durch den HNO-Facharzt geschaffen werden.

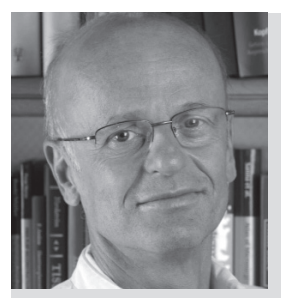

Prof. Dr. med. Dr. h.c. Friedrich Bootz ist Direktor der Klinik und Poliklinik für Hals-Nasen-Ohrenheilkunde/Chirurgie am Universitätsklinikum Bonn. E-Mail: friedrich.bootz@ukb.uni-bonn.de

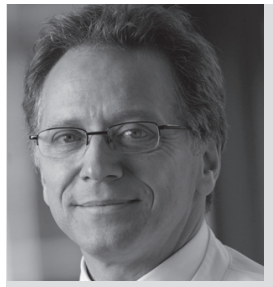

Prof. Dr. med. Dr. h.c. Peter K. Plinkert ist Direktor der Klinik und Poliklinik für Hals-Nasen-Ohrenheilkunde der Universität Heidelberg. E-Mail: peter.plinkert@med. uni-heidelberg.de

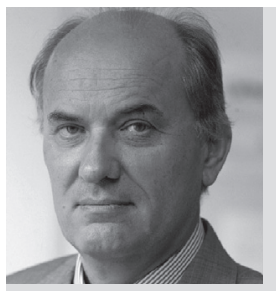

Prof. Dr. med. Dr. h.c. HansPeter Zenner ist Direktor der Universitäts-HNO-Klinik Tübingen. E-Mail: Hans-Peter.Zenner@med. uni-tuebingen.de

Interessenkonflikt Die Autoren erklären, dass keine Interessenkonflikte vorliegen.

\section{Literaturhinweis}

Teile dieses Artikels stammen aus folgendem Buch: Zenner H-P. Praktische Therapie von HNO-Krankheiten. 2. Aufl. Stuttgart: Schattauer; 2008, v. a. aus den Kapiteln

- Notfallmaßnahmen bei vitaler Bedrohung (Bootz F)

- Erkrankungen von Gesicht, Mittelgesicht und Rhinobasis: Verletzungen, thermische Schäden (Ambrosch P, Brunner FX) sowie Verletzungen, thermische/chemische Schäden (Dietz A, Brunner FX) und Nasenbluten / Epistaxis (Plinkert PK)

- Erkrankungen des Larynx: Entzündungen, Laryngopathien (Werner JA, Zenner H-P)

- Erkrankungen der Trachea: Entzündungen (Werner JA, Zenner H-P)

- Erkrankungen des Ösophagus: Verletzungen, chemische Schäden (Brunner FX, Plinkert PK)

\section{Weitere zitierte Literatur}

1 Wörth T. Management bei Luftnot - Akutmaßnahmen. Lege artis 2011; 1: 46-49

2 Zenner H-P, Hrsg. Praktische Therapie von HNO-Krankheiten. Stuttgart: Schattauer; 2008

3 Strutz J, Mann W, Hrsg. Praxis der HNO-Heilkunde, Kopf- und Halschirurgie. Stuttgart: Thieme; 2009

4 Secchi A, Ziegenfuß T. Checkliste Notfallmedizin. Stuttgart: Thieme; 2009

Beitrag online zu finden unter http://dx.doi.org/10.1055/s-0031-1295696 